AlltagsTrainingsProgramm

\section{Wenn die Tasche zum Trainingsgerät wird}

— Das AlltagsTrainingsProgramm (ATP) will Menschen ab 60 Jahren zu mehr Bewegung anregen. Das ATP wurde von der Bundeszentrale für gesundheitliche Aufklärung (BZgA) entwickelt. Es soll ab 2017 bundesweit in das Angebot von Sportvereinen aufgenommen werden. "Die Teilnehmer lernen, wie sie ihr Zuhause und ihre Umgebung zur Bewegungsförderung nutzen können - sei es durch Arm-Übungen mit Einkaufstaschen und anderen Alltagsgegenständen oder durch Muskel- und Gelenktraining beim Hausputz, beim Warten an der Bushaltestelle oder beim Stopp an der Parkbank", betont BZgA-Leiterin Dr. Heidrun Thaiss. Entwickelt wurde das ATP auf der Basis sportwissenschaftlicher Erkenntnisse und durch das Zentrum für Gesundheit durch Sport und Bewegung unter Leitung von Prof. Ingo Froböse evaluiert: „Die Teilnehmer waren zwischen 55 und 86 Jahre alt. Mehr als $80 \%$ gaben an, bislang nicht sportlich aktiv gewesen zu sein - genau die Zielgruppe des Programms. Eine hohe Teilnahmequote (75\%) und eine sehr gute Gesamtbewertung des AlltagsTrainingsProgramms sprechen für sich."

www.aelter-werden-in-balance.de

Welches Pflegeheim passt? "Heimhopping"

— Das Projekt "Heimhopping" hat die pensionierte Pflegewissenschaftlerin der Universität Witten/Herdecke, Prof. Dr. Angelika Zegelin entwickelt. „Teilnehmen kann jede und jeder, der - für sich oder andere - überlegt, in ein Heim zu gehen und nicht weiß, welche Kriterien wichtig sind", beschreibt sie den Ansatz. Es ginge darum, zu erkennen, ob das Angebot des Heims zur Biographie und Lebenseinstellung passe. Die erste Tour gab es am 15. Oktober in Dortmund.

www.uni-wh.de

\title{
Neue Studie
}

\section{Demenz \& Migrationshintergrund}

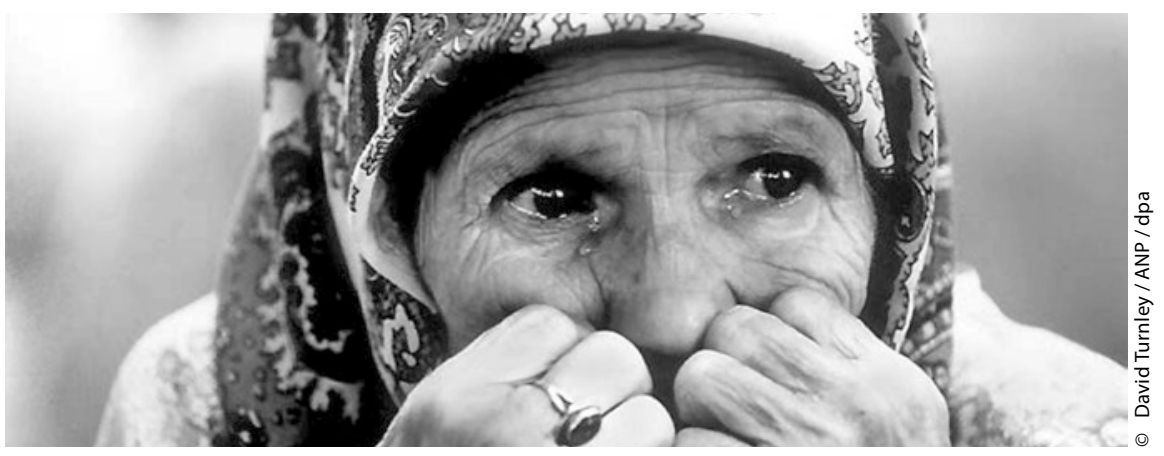

Über die spezielle Situation von Menschen mit Demenz in Migrantenfamilien ist bislang wenig bekannt.

— In Deutschland leben etwa 108.000 Menschen mit Migrationshintergrund mit unterschiedlichen dementiellen Veränderungen. Da über diese Gruppe wenig bekannt ist, werden jetzt unterschiedliche Weisen des Umgangs mit Demenz in Familien mit Migrationshintergrund näher untersucht. Die deutschlandweite Initiative Aktion Demenz e.V. führt das Vorhaben durch, unterstützt vom Gießener Institut für Soziologie.

Laut Projektleiterin Verena Rothe wolle man sich folgenden Fragen nähern: Wie wird Demenz verstanden? Wie sieht der Umgang mit Demenz aus und wer pflegt? Welche Kenntnisse und Wünsche über ambulante, institutionelle, nachbarschaftliche Hilfen gibt es? Welche kulturellen, familialen, traditionellen Orientierungen wirken sich wie aus? Gibt es Ansätze von demenzfreundlichen Gemeinschaften? Die Gießener Forscher führen vor allem Interviews mit Angehörigen von Menschen mit Demenz. Dabei werden sowohl Menschen mit unterschiedlichem Migrationshintergrund (z.B. Türkei, Osteuropa, Südeuropa) befragt als auch eine Art Kontrollgruppe ohne nennenswerten Migrationshintergrund. Erste Ergebnisse sollen Anfang 2017 vorliegen.

www.aktion-demenz.de

Service

\section{Digitaler Lesezirkel am HDZ NRW}

- Mit einer Neuigkeit für seine Patienten wartet das Herz- und Diabeteszentrum Nordrhein-Westfalen (HDZ NRW), Bad Oeynhausen, auf. Als erstes Klinikum in der Region bietet die Spezialklinik ein digitales Rezept gegen Langeweile an: Mit einer neuen Zeitungs-App können Patienten während ihres stationären Aufenthalts oder in den Wartebereichen der Ambulanzen mit ihrem Smartphone oder Tablet kostenlos auf mehr als 150 Zeitschriften zugreifen. „Wir wollen mit der Zeit gehen und setzen auf Informationen, die über unser hausweites und kostenloses Patienten-WLAN-Netz jederzeit verfügbar sind", betont Thomas Fehnker, stellvertretender Geschäftsführer des HDZ NRW. Notwendig seien lediglich ein Zugangs-Ticket und ein funktionsfähiges digitales Lesegerät.

Im Vorfeld hatten die IT-Abteilung und der Datenschutz des HDZ NRW sichergestellt, dass das Gesamtklinikum flächendeckend mit WLAN ausgeleuchtet ist und ein schneller Nutzerkomfort bei hoher Datensicherheit besteht. Bisher rund 1.000 Zugriffe auf den digitalen Lesezirkel belegen, dass sich die Investition in die Zeitungs-App lohnt. Sollte das Projekt weiterhin gut angenommen werden, zieht man eine Ausleihmöglichkeit von Tablets über die Patientenbibliothek in Erwägung.

www.hdz-nrw.de 